\title{
O efeito de reforço da nano-zircônia na resistência transversal da base de prótese acrílica reparada.
}

Carla Vanessa Rodrigues (1) e Bianca Ruiz Rufino (1)

\section{ARTIGO ORIGINAL}

Resumo

Objetivo. O objetivo deste estudo foi avaliar o efeito da incorporação de fibra de vidro, zircônia e nano-zircônia na resistência transversal de base de prótese dentária reparada. Materiais $e$ métodos. Oitenta espécimes de resina acrílica polimerizada termicamente foram preparados e divididos aleatoriamente em oito grupos: um grupo intacto (controle) e sete grupos reparados. Um grupo foi reparado com resina autopolimerizada enquanto os outros seis grupos foram reparados usando resina autopolimerizada reforçada com $2 \%$ em peso ou $5 \%$ em peso de fibra de vidro, zircônia ou partículas de nano-zircônia. Um teste de flexão de três pontos foi usado para medir a resistência transversal. Os resultados foram analisados usando SPSS e ANOVA de medida repetida e teste post hoc de mínima significância (LSD). Resultados. Entre os grupos reparados, verificou-se que a resina autopolimerizada reforçada com 2 ou 5\% em peso de nanozircônia apresentou a maior resistência transversal. Reparos com resina acrílica autopolimerizada reforçada com $5 \%$ em peso de zircônia apresentaram o menor valor de resistência transversal. Não houve diferença significativa entre os grupos reparados com resina de reparo sem reforço, $2 \%$ em peso de zircônia e resina reforçada com fibra de vidro. Conclusão. O reforço do material de reparo com nano-zircônia pode melhorar significativamente a resistência transversal de alguns polímeros de base de prótese dentária fraturada.

Palavras-chave: Nano-zircônia; Prótese; Resistência 


\title{
The reinforcing effect of nano-zirconia on the transverse strength of the repaired acrylic prosthesis base.
}

\begin{abstract}
Objective. The aim of this study was to evaluate the effect of incorporating fiberglass, zirconia and nano-zirconia on the transverse strength of the base of repaired dental prostheses. Materials and methods. Eighty specimens of thermally polymerized acrylic resin were prepared and randomly divided into eight groups: one intact group (control) and seven repaired groups. One group was repaired with self-cured resin while the other six groups were repaired using self-cured resin reinforced with $2 \%$ by weight or $5 \%$ by weight of fiberglass, zirconia or nanozirconia particles. A three-point bending test was used to measure transverse strength. The results were analyzed using SPSS and repeated measure ANOVA and post hoc test of least significance (LSD). Results.Among the repaired groups, it was found that the self-curing resin reinforced with 2 or $5 \%$ by weight of nano-zirconia had the highest transverse strength. Repairs with self-curing acrylic resin reinforced with $5 \%$ by weight of zirconia showed the lowest value of transverse strength. There was no significant difference between the groups repaired with unreinforced repair resin, $2 \%$ by weight of zirconia and fiberglass reinforced resin. Conclusion. Reinforcement of the repair material with nano-zirconia can significantly improve the transverse strength of some fractured dental prosthesis base polymers.
\end{abstract}

Keywords: Nano-zirconia; Prosthesis; Resistance

Instituição afiliada: 1- Acadêmicas do curso de Odontologia da Faculdade FAMA, Macapá, Amapá.

Dados da publicação: Artigo recebido em 10 de junho, revisado em 15 de junho, aceito para

publicação em 20 de junho e publicado em 30 de junho.

DOI: https://doi.org/10.36557/2674-8169.2021v3n6p06-29

Carla Vanessa Rodrigues c.rod.334@gmail.com

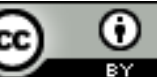

This work is licensed under a Creative Commons Attribution 4.0 International

License. 


\section{INTRODUÇÃO}

A fratura da prótese é um problema comum na prática da prótese dentária que incomoda tanto os pacientes quanto os protodontistas. Forças mastigatórias excessivas ou deformação da dentadura durante o uso podem resultar em forças de flexão que contribuem para a fadiga do material e subsequente fratura [ 1 ]. Uma nova construção de prótese aumenta o custo e é demorada, então o reparo da prótese é preferível [ 2 ]. O reparo satisfatório deve ser fácil e rápido e combinar com a cor original da base da prótese, mantendo a precisão dimensional [ 3 ]. O reparo de próteses depende de muitas variáveis, incluindo tipo de material, reforço de material, design de superfície e tratamento de superfície [2] Vários materiais têm sido usados para reparar bases de dentaduras fraturadas, incluindo resina acrílica autopolimerizada, polimerizada por luz visível, polimerizada por calor ou por micro-ondas [ 4 , 5 ]. A maioria (86\%) dos reparos da base da prótese é feita com resina acrílica autopolimerizada [ 6 ], pois é fácil de manipular e rápida e pode ser usada na cadeira [ 7 ]. Infelizmente, foi demonstrado que sua resistência varia de 18 a $81 \%$ da resina de prótese dentária polimerizada por calor intacta [ 3,8$]$ Muitas tentativas têm sido feitas para superar essa lacuna por meio do uso de material de reparo reforçado e / ou modificação do design e tratamento da superfície de reparo. Hanna et al. investigaram o efeito do chanfro de $45^{\circ}$ da superfície de reparo na resistência transversal da base da prótese reparada e descobriram que valores mais elevados foram obtidos [ 9 ]. O biselamento da superfície de reparo mudou o tipo de fratura de adesivo fraco para fratura coesiva forte [ 10 ]. É apropriado tratar a superfície de reparo com monômero de reparo, pois modifica a estrutura da superfície e aumenta sua ligação ao material de reparo [ 11 - 13 ].

A fibra de vidro é um dos materiais de reforço mais comuns e muitas investigações sobre seu efeito na base da prótese dentária reparada foram realizadas. A adição de fibra de vidro ao material de reparo melhora a resistência do reparo da base da prótese e pode diminuir a ocorrência de fraturas futuras [ $3,14,15$ ]. Isso pode ser atribuído ao fato da fibra de vidro possuir uma alta resiliência o que permite que as tensões sejam recebidas por elas sem deformação [ 16 ].

Zircônia (ZrO 2 ) é um óxido de metal e pode ser usado como um material de reforço para melhorar a resistência transversal da resina para base de próteses [ 17,18 ]. O reforço da base da prótese acrílica com zircônia aumenta significativamente sua resistência transversal [ 19 ]. Recentemente, a nanotecnologia invadiu o campo da prótese dentária para fins médicos e de aprimoramento de materiais. As propriedades da resina reforçada por nanopartículas dependem do tamanho, forma, tipo e concentração das partículas adicionadas [ 20 ]. Adições de nano-zircônia à base de dentadura de polimetilmetacrilato (PMMA) aumentam a resistência transversal devido 
ao seu pequeno tamanho e distribuição homogênea [ 21 ].

A desvantagem dos materiais de reparo comumente usados é que eles têm pouca resistência. A pesquisa atual na área de materiais odontológicos está focada em encontrar o material de reparo apropriado com resistência adequada e vida útil prolongada. Até o momento, o efeito do nano-ZrO 2 na resistência do reparo não foi investigado. Portanto, este estudo foi conduzido para avaliar o efeito de reforço de diferentes concentrações de fibra de vidro, zircônia e nano-zircônia na resistência transversal de uma base de prótese dentária reparada. A hipótese nula era que a adição de diferentes concentrações de zircônia ou nano-zircônia não melhoraria a resistência transversal da base da prótese reparada.

\section{MATERIAIS E MÉTODOS}

De acordo com a especificação ANSI / ADA número 12, oitenta espécimes retangulares de resina acrílica polimerizada por calor com dimensões $(65 \times 10 \times 2,5 \mathrm{~mm}$ $\pm 0,1$ ) foram preparados usando moldes customizados [22]. Os moldes foram encerados (Cavex Set Up Wax, Cavex, Holanda) e, em seguida, os padrões de cera foram investidos em gesso dentário tipo III (GC Fujirock EP, Bélgica) dentro de um frasco (61B Two Flask Compress, Handler Manufacturing, EUA) e depois desparafinados para crie o espaço do molde. De acordo com as instruções do fabricante, resina acrílica polimerizada por calor (Major Base 20, Major Prodotti Dentari SPA, Itália) foi misturada e embalada na fase de massa na cavidade do molde e o fechamento do teste foi feito e, em seguida, o frasco foi fechado e mantido sob pressão de bancada por 30 minutos. Frasco com espécimes de resina acrílica foi processado por 8 horas em banho-maria a $74^{\circ} \mathrm{C}$ e então a temperatura foi aumentada para $100^{\circ} \mathrm{C}$ por 1 hora em unidade de cura térmica (KaVo Elektrotechnisches Werk GmbH, D-88299, Alemanha).

Após a cura, os frascos foram resfriados em bancada até a temperatura ambiente antes da remoção do revestimento. $O$ excesso de resina dos espécimes desincluídos foi removido com uma broca de carboneto de tungstênio (HM251 FX 040 HP, Meisinger, EUA), polido com polidor de acrílico (HM251FX-060, Meisinger, EUA) e, em seguida, armazenado em água destilada a $37^{\circ} \mathrm{C}$ por 48 horas. Todos os espécimes foram divididos aleatoriamente em oito grupos: um intacto e sete grupos reparados (Tabela 1). Para criar uma lacuna de reparo de $3 \mathrm{~mm}$, os espécimes de reparo foram colocados no molde e numerados em ambas as extremidades para remontagem. A marca foi desenhada no centro da amostra e, em seguida, a 1,5 mm de distância dessa marca, duas linhas foram traçadas em ambos os lados e perpendiculares à borda longa da amostra. Essas duas linhas foram estendidas nas superfícies do molde como um guia para todos os espécimes. Nessas linhas, os espécimes foram cortados com disco de diamante de baixa velocidade (DeguDent, GmbH, REF 59903107, Dentsply, Alemanha) sob irrigação abundante. A junta chanfrada de $45^{\circ}$ padronizada foi preparada medindo 
2,5 mm e desenhando uma linha paralela à aresta preparada. Da mesma forma, os lados do molde foram cortados no centro medindo $8 \mathrm{~mm}$ da superfície superior e $3 \mathrm{~mm}$ da superfície inferior preservando a base do molde intacta. As amostras foram colocadas no molde e cortadas na direção do chanfro por disco de diamante guiado por linhas e superfícies do molde para criar uma lacuna de reparo de $3 \mathrm{~mm} \times 10 \mathrm{~mm} \times 2,5 \mathrm{~mm}$ com uma junta chanfrada de $45^{\circ}$. Fibra de vidro (vidro E; comprimento $=3 \mathrm{~mm}$, Shanghai Richem International Co., Ltd., China), zircônia (99,5\%, 5 um, 1314-23-4, Shanghai Richem International Co., Ltd., China), e pó de nano-zircônia $(99,9 \%,<100$ nm, 1314-234, Shanghai Richem International Co., Ltd., China) foram pesados usando uma balança eletrônica (S-234; Denver Instrument, Alemanha) em uma concentração de $2 \%$ em peso e $5 \%$ em peso de pó de resina acrílica autopolimerizada (Major Repair; Major Prodotti Dentari SPA, Itália). Fibra de vidro pré-pesada, zircônia e pó de nano-zircônia foram adicionados separadamente ao pó de resina acrílica autopolimerizada e completamente misturados usando um almofariz e pilão para obter uma distribuição igual de partículas e cor uniforme.

De acordo com a numeração, as seções de amostra foram remontadas no molde original e fixadas criando $3 \mathrm{~mm}$ entre as seções remontadas. As superfícies de reparo foram tratadas com o monômero de metacrilato de metila por três minutos. O reparo foi feito usando o método de polimerização de monômero-polímero e preenchendo ligeiramente a lacuna de reparo para compensar a contração de polimerização e os procedimentos de acabamento. Uma vez que a superfície do material de reparo perdeu seu vidrado, os moldes e seus conteúdos foram colocados na câmara de pressão contendo água a $\left(40^{\circ} \mathrm{C}\right.$ ) e a uma pressão de 30 IB / polegada2 (libra-força por polegada quadrada) por 15 minutos.

Após a cura, os corpos-de-prova foram retirados do molde, acabados, polidos e, em seguida, colocados em água destilada e incubados a $37^{\circ} \mathrm{C}$ por 48 horas e testados $[3,23]$. Para determinar a resistência transversal, a carga de fratura foi medida usando o teste de flexão de três pontos em uma máquina de teste universal (INSTRON 8871, sistema servo hidráulico, software Merlin 2). Os corpos-de-prova foram colocados em um aparelho de flexão de 3 pontos e o vão de apoio foi de $50 \mathrm{~mm}$. A carga foi aplicada no ponto médio da área reparada com velocidade da cruzeta de $5 \mathrm{~mm} / \mathrm{min}$ até que o

espécime fraturou e a carga de fratura foi registrada. A fórmula $\left(\mathrm{TS}=\frac{3 W L}{2 b d^{2}}\right)$ foi usado para calcular os valores de resistência transversal de cada corpo de prova, onde é a resistência transversal (em $\mathrm{MPa}$ ), é a carga de fratura (N), é a distância entre os dois suportes, é a largura do corpo de prova e é a espessura do corpo de prova [24 ,25].

Tabela1: Grupos testados e codificação de acordo com o reforço do material de reparo. 


\begin{tabular}{lr} 
Código de grupo & Material de reparo \\
\hline HC & Amostras intactas polimerizadas por calor (controle) \\
AP & Resina acrílica autopolimerizada \\
$2 \mathrm{GF}$ & Resina acrílica autopolimerizada reforçada com $2 \%$ em peso de fibra de vidro \\
$5 \mathrm{GF}$ & Resina acrílica autopolimerizada reforçada com $5 \%$ em peso de fibra de vidro \\
$2 Z \mathrm{R}$ & Resina acrílica autopolimerizada reforçada com $2 \%$ em peso de zircônia \\
$5 Z \mathrm{R}$ & Resina acrílica autopolimerizada reforçada com $5 \%$ em peso de zircônia \\
$2 \mathrm{NZR}$ & Resina acrílica autopolimerizada reforçada com $2 \%$ em peso de nano-zircônia \\
$5 \mathrm{NZR}$ & Resina acrílica autopolimerizada reforçada com $5 \%$ em peso de nano-zircônia
\end{tabular}

\section{ANÁLISE ESTATÍSTICA}

A análise dos dados foi realizada por meio do software SPSS-20.0 da IBM, Chicago (EUA). Os resultados foram apresentados como média e desvio padrão. ANOVA de medida repetida foi aplicada para ver a significância estatística das variáveis em comparação com o grupo de controle e AP. O teste de significância mínima post hoc (LSD) foi usado para ver a comparação entre pares das variáveis. valor $\leq 0,05$ foi considerado resultado estatisticamente significativo.

\section{RESULTADOS}

O valor médio e o desvio padrão da resistência transversal estão resumidos na Tabela 2 . A análise estatística revelou que a força transversal do HC foi o maior valor de força entre os grupos testados (Figura 2 ). Houve diferenças estatisticamente significativas na força transversal entre os grupos reparados 5NZR, 2NZR, 2GF e 5ZR em comparação com AP ( ). Os maiores valores de força transversal foram nos grupos 5 NZR, 2NZR e 2GF, respectivamente. Enquanto isso, 5ZR mostrou uma diminuição significativa no valor da força transversal. Não houve diferença significativa na força transversal entre $2 Z R$ e $5 G F$ com AP.

Tabela 2: Média, desvio padrão (DP) e valores para diferentes concentrações de reforço de fibra de vidro, zircônia e nano-zircônia. 


\begin{tabular}{lccc}
\hline & Média \pm SD & Versus HC & Versus AP \\
\hline HC, controle & $83,01 \pm 3,03$ & - & - \\
AP & $44,85 \pm 3,68$ & - & - \\
$2 \mathrm{GF}$ & $56,98 \pm 2,58^{* *}$ & 0,0001 & 0,001 \\
$5 \mathrm{GF}$ & $42,75 \pm 2,45^{*}$ & 0,0001 & 0,175 \\
2ZR & $50,07 \pm 2,97^{*}$ & 0,0001 & 0,064 \\
$5 \mathrm{ZR}$ & $40,21 \pm 3,31^{* *}$ & 0,0001 & 0,035 \\
2NZR & $65,43 \pm 2,62^{* *}$ & 0,001 & 0,0001 \\
$5 \mathrm{NZR}$ & $70,77 \pm 2,80^{* *}$ & 0,001 & 0,0001 \\
\hline
\end{tabular}

\footnotetext{
"Significância estatística do material com grupo controle apenas em. Significância estatística do material com controle, bem como AP em $. P \leq 0.05$
} $* P \leq 0.05$

Este estudo in vitro foi realizado para avaliar o efeito de reforço de diferentes concentrações de fibra de vidro, zircônia e nano-zircônia na resistência transversal de uma base de prótese dentária reparada. Os resultados revelaram que o grupo HC apresentou os maiores valores de força transversal entre todos os grupos, o que está de acordo com os resultados de um estudo anterior [26]. Alguns espécimes reparados reforçados exibiram um aumento na resistência transversal em comparação com AP; portanto, a hipótese nula foi rejeitada. A força transversal do AP diminuiu até a metade do valor do grupo $\mathrm{HC}$, o que está de acordo com os resultados de estudos anteriores [ $3,27,28]$ A diminuição da resistência transversal pode ser devido à menor resistência da resina acrílica autopolimerizada; processo de polimerização insuficiente; e o monômero residual retido no local do reparo [ 29 - 31 ] (Figura 1 ).

A adição de fibra de vidro ao material de reparo foi encontrada para melhorar a resistência transversal da base da prótese reparada e pode ser mais aceitável para uso devido à estética e facilidade de uso [ 32 ]. Os resultados do estudo atual revelaram um aumento na força transversal de 2GF em comparação com AP, o que está de acordo com os resultados de um estudo anterior [ 32] Esse aumento pode ser atribuído ao fato da fibra de vidro possuir uma alta resiliência o que permite que as tensões sejam recebidas por elas sem deformação permanente [ 16 ]. O 5GF mostrou uma diminuição na força transversal, o que está de acordo com um estudo anterior [ 33 ]. Isso pode ser explicado devido ao alto teor de fibra que pode afetar a resistência de união entre o material de reparo e a base da prótese [34]

Os resultados deste estudo mostraram que a adição de $2 Z R$ melhorou a resistência transversal dos corpos de prova reparados. Este aumento na resistência transversal pode ser resultante da transformação da zircônia da fase tetragonal para monoclínica, resultando na absorção da energia de propagação da trinca em um 
processo denominado tenacificação por transformação. Além disso, neste processo, a expansão dos cristais de $\mathrm{ZrO}_{2}$ ocorre e coloca a trinca sob um estado de tensão compressiva e interrompendo a propagação da trinca [ 35].

Os resultados do presente estudo mostraram que a força transversal diminuiu proporcionalmente à concentração de zircônia. As adições de 5ZR resultaram em uma diminuição significativa na resistência transversal em comparação com AP. Esta redução na resistência transversal pode ser causada por muitos motivos, incluindo maior porcentagem de enchimento que resultou em mais defeitos que afetam a resistência do material; agrupamento das partículas dentro da resina; e mais partículas de enchimento após atingir a saturação da matriz leva à interrupção na continuidade da matriz da resina [ 36,37 ]. Em contraste, um estudo relatou que a resistência transversal aumentou com o aumento do conteúdo de zircônia [ 19 ]. Os resultados deste estudo mostraram que a resistência transversal aumentou significativamente após a incorporação de 2NZR [38 ].

Este aumento na resistência transversal pode ser devido à boa distribuição das partículas nanométricas que lhes permitem entrar e preencher os espaços entre as cadeias poliméricas, resultando em aumento da resistência ao cisalhamento interfacial entre as nanopartículas e as cadeias poliméricas que melhoram a resistência transversal [ 39 ]. Também foi observado que a força transversal máxima foi registrada com 5NZR [ 21,39 ], e o aumento na porcentagem de nano-zircônia aumenta a força transversal, o que está de acordo com um estudo anterior [ 39 ], embora esteja em desacordo com outros estudos [ 21,40$]$.

A implicação clínica do presente estudo é que a incorporação de nano-zircônia em resina de reparo autopolimerizada aumenta a resistência da base da prótese reparada. O desenho do estudo não pode imitar as condições clínicas; portanto, essa limitação afetou os procedimentos de teste e as propriedades mecânicas investigadas. Pesquisas futuras para estudar esses materiais devem se concentrar na simulação de condições clínicas com as próteses existentes e na implementação de testes apropriados.

Imagem 1: Amostra de resina acrílica carregada em máquina de teste universal e sujeita a carga de fratura. 

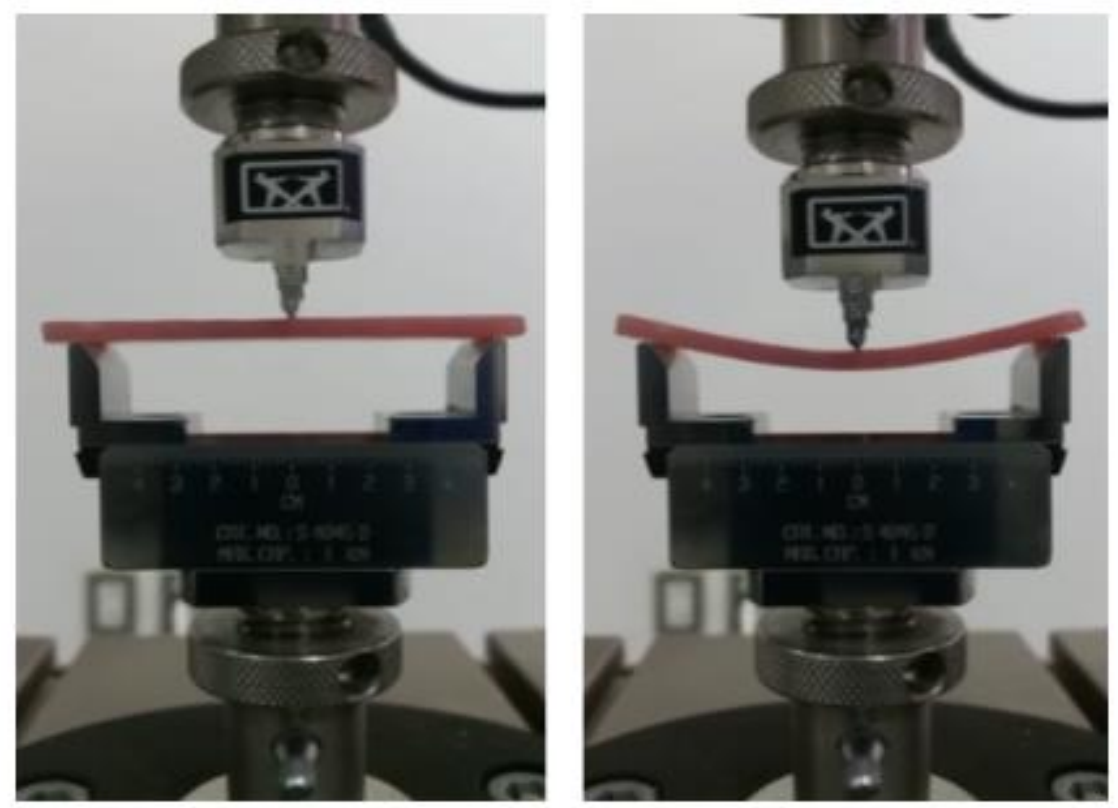

Imagem 2: Valor médio da resistência transversal para todos os grupos testados.

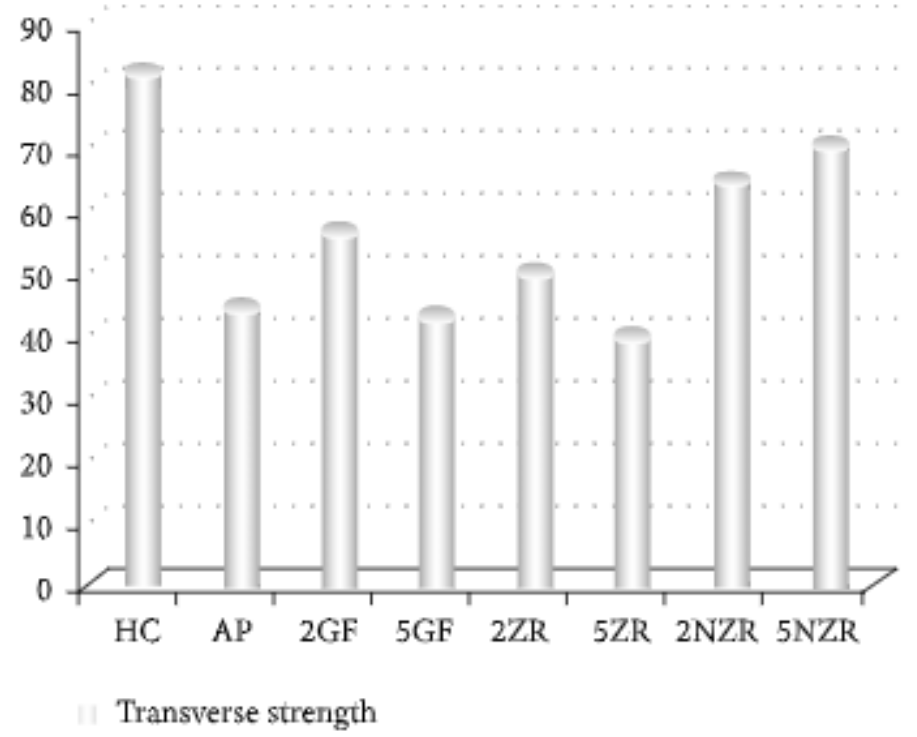

\section{CONCLUSÃO}

De acordo com os resultados e as limitações deste estudo in vitro, pode-se concluir que a nano-zircônia pode ser considerada uma nova abordagem para o reparo de bases de próteses. Os reparos resultaram em uma resistência transversal significativamente maior em comparação com a resina reparada não reforçada. 


\section{THE AUTHORS DECLARE NO CONFLICTS OF INTEREST.}

\section{DECLARAÇÃO CCBY}

Este artigo é uma cópia com adaptação para o português do original “Gad, Mohammed, et al. "The reinforcement effect of nano-zirconia on the transverse strength of repaired acrylic denture base." International journal of dentistry 2016 (2016)." 41

Esta cópia com adaptação para o português teve como alteração além do idioma a autoria do artigo.

Os autores da publicação original não deram endosso específico a esta cópia com adaptação para a português, a não ser a licença CCBY 4.0 disponibilizada pelo periódico que publicou o artigo original.

Este é o link da licença: https://creativecommons.org/licenses/by/4.0/

Este é o link do artigo original: https://www.hindawi.com/journals/ijd/2016/7094056/

\section{REFERÊNCIAS}

1. U. R. Darbar, R. Huggett, and A. Harrison, "Denture fracture: a survey," British Dental Journal, vol. 176, no. 9, pp. 342-345, 1994.View at: Publisher Site I Google Scholar

2. R. S. Seó, K. H. Neppelenbroek, and J. N. A. Filho, "Factors affecting the strength of denture repairs: topics of interest," Journal of Prosthodontics, vol. 16, no. 4, pp. 302-310, 2007.View at: Publisher Site | Google Scholar

3. G. L. Polyzois, P. A. Tarantili, M. J. Frangou, and A. G. Andreopoulos, "Fracture force, deflection at fracture, and toughness of repaired denture resin subjected to microwave polymerization or reinforced with wire or glass fiber," Journal of Prosthetic Dentistry, vol. 86, no. 6, pp. 613-619, 2001.View at: Publisher Site | Google Scholar

4. J. N. Arioli Filho, L. E. Butignon, R. D. P. Pereira, M. G. Lucas, and F. D. A. Mollo Jr., "Flexural strength of acrylic resin repairs processed by different methods: water bath, microwave energy and chemical polymerization," Journal of Applied Oral Science, vol. 19, no. 3, pp. 249-253, 2011.View at: Google Scholar

5. S. Suvarna, T. Chhabra, D. Raghav, D. Singh, P. Kumar, and S. Sahoo, "Residual monomer content of repair autopolymerizing resin after microwave postpolymerization treatment," European Journal of Prosthodontics, vol. 2, no. 1, pp. 28-32, 2014.View at: $\underline{\text { Publisher Site I Google Scholar }}$

6. A. I. Zissis, G. L. Polyzois, and S. A. Yannikakis, "Repairs in complete dentures: results of a survey," Quintessence of Dental Technology, vol. 20, pp. 149-155, 1997.View at: Google 


\section{$\underline{\text { Scholar }}$}

7. C. Bural, G. Bayraktar, I. Aydin, I. Yusufoğlu, N. Uyumaz, and M. Hanzade, "Flexural properties of repaired heat-polymerising acrylic resin after wetting with monomer and acetone," Gerodontology, vol. 27, no. 3, pp. 217-223, 2010.View at: $\underline{\text { Publisher }}$ Site I Google Scholar

8. I. Kostoulas, V. T. Kavoura, M. J. Frangou, and G. L. Polyzois, "Fracture force, deflection, and toughness of acrylic denture repairs involving glass fiber reinforcement," Journal of Prosthodontics, vol. 17, no. 4, pp. 257-261, 2008.View at: $\underline{\text { Publisher Site I Google Scholar }}$

9. E. A. Hanna, F. K. Shah, and A. A. Gebreel, "Effect of joint surface contours on the transverse and impact strength of denture base resin repaired by various methods: an in vitro study," Journal of American Science, vol. 6, no. 9, pp. 115-125, 2010.View at: Google Scholar

10. S. Kirti, M. R. Dhakshaini, and A. K. Gujjari, "Evaluation of transverse bond strength of heat cured acrylic denture base resin repaired using heat polymerizing, autopolymerizing and fiber reinforced composite resin-an in vitro study," International Journal of Clinical Cases and Investigations, vol. 4, no. 2, pp. 33-43, 2012.View at: Google Scholar

11. P. K. Vallittu, V. P. Lassila, and R. Lappalainen, "Wetting the repair surface with methyl methacrylate affects the transverse strength of repaired heat-polymerized resin," The Journal of Prosthetic Dentistry, vol. 72, no. 6, pp. 639-643, 1994.View at: $\underline{\text { Publisher }}$ Site I Google Scholar

12. M. Vojdani, S. Rezaei, and L. Zareeian, "Effect of chemical surface treatments and repair material on transverse strength of repaired acrylic denture resin," Indian Journal of Dental Research, vol. 19, no. 1, pp. 2-5, 2008.View at: $\underline{\text { Publisher Site I Google Scholar }}$

13. H. Minami, S. Suzuki, Y. Minesaki, H. Kurashige, and T. Tanaka, "In vitro evaluation of the influence of repairing condition of denture base resin on the bonding of autopolymerizing resins," Journal of Prosthetic Dentistry, vol. 91, no. 2, pp. 164-170, 2004.View at: Publisher Site I Google Scholar

14. H. D. Stipho, "Repair of acrylic resin denture base reinforced with glass fiber," The Journal of Prosthetic Dentistry, vol. 80, no. 5, pp. 546-550, 1998.View at: $\underline{\text { Publisher Site I Google }}$ Scholar

15. E. Nagai, K. Otani, Y. Satoh, and S. Suzuki, "Repair of denture base resin using woven metal and glass fiber: effect of methylene chloride pretreatment," The Journal of Prosthetic Dentistry, vol. 85, no. 5, pp. 496-500, 2001.View at: $\underline{\text { Publisher Site I Google Scholar }}$

16. G. Uzun, N. Hersek, and T. Tinçer, "Effect of five woven fiber reinforcements on the impact and transverse strength of a denture base resin," The Journal of Prosthetic Dentistry, vol. 
81, no. 5, pp. 616-620, 1999.View at: $\underline{\text { Publisher Site | Google Scholar }}$

17. N. V. Asar, H. Albayrak, T. Korkmaz, and I. Turkyilmaz, "Influence of various metal oxides on mechanical and physical properties of heat-cured polymethylmethacrylate denture base resins," Journal of Advanced Prosthodontics, vol. 5, no. 3, pp. 241-247, 2013.View at: Publisher Site I Google Scholar

18. A. O. Alhareb and Z. A. Ahmad, "Effect of $\mathrm{Al}_{2} \mathrm{O}_{3} / \mathrm{ZrO}_{2}$ reinforcement on the mechanical properties of PMMA denture base," Journal of Reinforced Plastics and Composites, vol. 30, no. 1, pp. 86-93, 2011.View at: Publisher Site I Google Scholar

19. N. M. Ayad, M. F. Badawi, and A. A. Fatah, "The effect of reinforcement of high-impact acrylic resin with zirconia on some physical and mechanical properties," Cairo Dental Journal, vol. 24, no. 2, pp. 245-250, 2008. View at: Google Scholar

20. I. N. Safi, "Evaluation the effect of nano-fillers $\left(\mathrm{TiO}_{2}, \mathrm{AL}_{2} \mathrm{O}_{3}, \mathrm{SiO}_{2}\right)$ addition on glass transition temperature, E-moudulus and coefficient of thermal expansion of acrylic denture base material," Journal of Baghdad College of Dentistry, vol. 26, no. 1, pp. 37-41, 2014.View at: Publisher Site I Google Scholar

21. N. S. Ihab and M. Moudhaffar, "Evaluation the effect of modified nano-fillers addition on some properties of heat cured acrylic denture base material," Journal of Baghdad College of Dentistry, vol. 23, no. 3, pp. 23-29, 2011.View at: Google Scholar

22. American Dental Association, "Revised American Dental Association Specification no. 12 for denture base polymers," Journal of the American Dental Association, vol. 90, no. 2, pp. 451-458, 1975.View at: Publisher Site I Google Scholar

23. R. N. Rached, J. M. Powers, and A. A. Del Bel Cury, "Efficacy of conventional and experimental techniques for denture repair," Journal of Oral Rehabilitation, vol. 31, no. 11, pp. 1130-1138, 2004.View at: Publisher Site I Google Scholar

24. M. Alkurt, Z. Yeşil Duymuş, and M. Gundogdu, "Effect of repair resin type and surface treatment on the repair strength of heat-polymerized denture base resin," Journal of Prosthetic Dentistry, vol. 111, no. 1, pp. 71-78, 2014.View at: Publisher Site I Google Scholar

25. R. Zbigniew and D. Nowakowska, "Mechanical properties of hot curing acrylic resins after reinforced with different kinds of fibers," International Journal of Biomedical Materials Research, vol. 1, no. 1, pp. 9-13, 2013.View at: $\underline{\text { Publisher Site I Google Scholar }}$

26. F. Keyf and G. Uzun, "The effect of glass fibre-reinforcement on the transverse strength, deflection and modulus of elasticity of repaired acrylic resins," International Dental Journal, vol. 50, no. 2, pp. 93-97, 2000.View at: Publisher Site I Google Scholar

27. H. D. Stipho and A. S. Stipho, "Effectiveness and durability of repaired acrylic resin 
joints," The Journal of Prosthetic Dentistry, vol. 58, no. 2, pp. 249-253, 1987.View at: $\underline{\text { Publisher Site I Google Scholar }}$

28. F. Faot, W. J. da Silva, R. S. da Rosa, A. A. Del Bel Cury, and R. C. M. R. Garcia, "Strength of denture base resins repaired with auto- and visible light-polymerized materials," Journal of Prosthodontics, vol. 18, no. 6, pp. 496-502, 2009.View at: Publisher Site I Google Scholar

29. R. N. Rached, J. M. Powers, and A. A. Del Bel Cury, "Repair strength of autopolymerizing, microwave, and conventional heat-polymerized acrylic resins," Journal of Prosthetic Dentistry, vol. 92, no. 1, pp. 79-82, 2004.View at: $\underline{\text { Publisher Site I Google Scholar }}$

30. P. K. Vallittu, "The effect of surface treatment of denture acrylic resin on the residual monomer content and its release into water," Acta Odontologica Scandinavica, vol. 54, no. 3, pp. 188-192, 1996.View at: $\underline{\text { Publisher Site I Google Scholar }}$

31. J. A. Kenneth, C. Shen, and H. R. Rawls, Phillips' Science of Dental Materials, Elsevier Saunders, Philadelphia, Pa, USA, 12th edition, 2013.

32. G. S. Solnit, "The effect of methyl methacrylate reinforcement with silane-treated and untreated glass fibers," The Journal of Prosthetic Dentistry, vol. 66, no. 3, pp. 310-314, 1991.View at: Publisher Site I Google Scholar

33. N. Anasane, Y. Ahirrao, D. Chitnis, and S. Meshram, "The effect of joint surface contours and glass fiber reinforcement on the transverse strength of repaired acrylic resin: an in vitro study," Dental Research Journal, vol. 10, no. 2, pp. 214-219, 2013.View at: Publisher Site I Google Scholar

34. M. M. Gad, M. A. Helal, M. E. Abdel-Nasser, and M. I. Seif-Elnassr, "Effect of the microwave and reinforcement of repaired acrylic resin on some mechanical properties," Al-Azhar Journal of Dental Science, vol. 12, no. 1, pp. 65-72, 2009.View at: Google Scholar

35. J. A. Kenneth, Phillips' Science of Dental Materials, Saunders, Philadelphia, Pa, USA, 11th edition, 2003.

36. T. Nejatian, A. Johnson, and R. Van Noort, "Reinforcement of denture base resin," Advances in Science and Technology, vol. 49, pp. 124-129, 2006.View at: $\underline{\text { Publisher }}$ Site I Google Scholar

37. M. Braden, "Some aspects of the chemistry and physics of dental resins," Advances in Dental Research, vol. 2, no. 1, pp. 93-97, 1988.View at: Google Scholar

38. X.-J. Zhang, X.-Y. Zhang, B.-S. Zhu, and C. Qian, "Effect of nano $\mathrm{ZrO}_{2}$ on flexural strength and surface hardness of polymethylmethacrylate," Shanghai Kou Qiang Yi Xue, vol. 20, no. 4, pp. 358-363, 2011.View at: Google Scholar 
39. M. A. Ahmed and M. I. Ebrahim, "Effect of zirconium oxide nano-fillers addition on the flexural strength, fracture toughness, and hardness of heat-polymerized acrylic resin," World Journal of Nano Science and Engineering, vol. 4, no. 2, pp. 50-57, 2014.View at: $\underline{\text { Publisher Site I Google Scholar }}$

40. H. K. Hameed and H. A. Rahman, "The effect of addition nano particle ZrO2 on some properties of autoclave processed heat cure acrylic denture base material," Journal of Baghdad College of Dentistry, vol. 27, no. 1, pp. 32-39, 2015.View at: $\underline{\text { Publisher }}$ Site I Google Scholar

41. Gad, Mohammed, et al. "The reinforcement effect of nano-zirconia on the transverse strength of repaired acrylic denture base." International journal of dentistry 2016 (2016). 